\title{
Simulation of choroidal neovascularization in rabbits using indirect transpupillary laser coagulation
}

\author{
E.V. Prudnikova ${ }^{1, *}, A . E$. Katelnikova ${ }^{2}$, and $Z h . Y u$. Ustenko ${ }^{2}$ \\ ${ }^{1}$ FSBEI SPbSUVM, Saint Petersburg, Russia \\ ${ }^{2}$ JSC "NPO "HOUSE OF PHARMACY", Saint Petersburg, Russia
}

\begin{abstract}
Objective: to identify the applicability of indirect transpupillary laser coagulation (ITLC) for modeling choroidal neovascularization $(\mathrm{CN})$ in rabbits and its optimal parameters.

Materials and methods: 12 rabbits, divided into 2 equal groups, were subjected to single- and double-sided ITLC, respectively, using a laser device with a wavelength of $810 \mathrm{~nm}$ (using different powers), an indirect ophthalmoscope and a lens. The time spent on coagulation, ophthalmoscopic signs on the 1st, 8th, 15th, 22nd and 29th days of the study, the severity of CN (histological and immunohistochemical (IHC) studies), body weight and general condition of the animals were also evaluated. Results: in all cases, it was possible to apply the coagulants using ITLC. During ophthalmoscopy, peripheral vascularization was detected on the 8th-29th day. Histological and IHC studies revealed significant tissue damage at a power of $700,1000 \mathrm{~mW}$, as well as signs of $\mathrm{CN}$ when using a power of $500 \mathrm{~mW}$ on the 15 th day of the study.

Conclusion: ITLC is a safe method and can be used to simulate $\mathrm{CN}$ in rabbits at a power of no more than $500 \mathrm{~mW}$.
\end{abstract}

\section{Introduction}

A reliable method for choroidal neovascularization simulation in animals is required to study the effect of vascular endothelial growth factor (VEGF) inhibitors used in the treatment of age-related macular degeneration (AMD), and consists in the rupture of the Bruch membrane by laser coagulation [1]. The first model of experimental choroidal neovascularization in animals was developed by Ryan et al. in non-human primates [2]. In the available literature, mice, rats, monkeys, and rabbits are used as test systems for this model pathology [3-11], however, there is no data on the use of indirect transpupillary laser coagulation (in all cases, the slit lamp method was used), and therefore the purpose of the study was to evaluate the applicability of indirect transpupillary laser coagulation for choroidal neovascularization simulation in rabbits and to identify its optimal parameters.

\footnotetext{
* Corresponding author: vasilyeva.eye@yandex.ru
} 


\section{Materials and methods}

Animals: the study included 12 male rabbits of the Sovetskaya Chinchilla breed, aged 5 months. The average body weight is $4215 \pm 132 \mathrm{~g}$. The animals were kept in individual cages, received mixed feed for rabbits and water. The animals were vaccinated and treated for ecto -and endoparasites.

The characteristics of the studied groups and the list of manipulations are presented in Tables 1 and 2.

Table 1. Characteristics of the studied groups

\begin{tabular}{|c|c|c|c|c|c|c|}
\hline \multirow{2}{*}{$\begin{array}{c}\text { Gr } \\
\text { ou } \\
\mathrm{p}\end{array}$} & Group description & \multirow{2}{*}{$\begin{array}{c}\text { Quantity } \\
\text { of animals }\end{array}$} & \multicolumn{4}{|c|}{ Euthanasia day, animal numbers } \\
\cline { 4 - 7 } & & 8th & 15th & 22nd & 29th \\
\hline 1 & $\begin{array}{c}\text { Induction of one eye } \\
\text { pathology }\end{array}$ & 6 & $1.1-1.3$ & - & $1.4-1.6$ & - \\
\hline 2 & $\begin{array}{c}\text { Induction of both eyes' } \\
\text { pathology }\end{array}$ & 6 & - & $2.1-2.3$ & - & $2.4-2.6$ \\
\hline
\end{tabular}

Table 2. List of manipulations

\begin{tabular}{|c|c|}
\hline Manipulations & Days of the experiment \\
\hline Induction of pathology & 1 st \\
\hline Registration of animal body weight & 1st, 8th, 15th, 22nd, 29th \\
\hline Clinical observation & daily \\
\hline Ophthalmoscopy & $\begin{array}{c}\text { 1st (before pathology formation), } \\
\text { 8th, 15th, 22nd, 29th }\end{array}$ \\
\cline { 1 - 2 } Euthanasia & In accordance with table 1 \\
\hline $\begin{array}{c}\text { Histological/immunohistochemical } \\
\text { examination of the retina }\end{array}$ & \\
\hline
\end{tabular}

\subsection{Induction of pathology}

Before the induction of pathology, rabbits were instilled with 1 drop of $1 \%$ Tropicamide (S.C. Rompharm Company S.R.L., Romania) in the conjunctival sac. Next, the anesthesia procedure was performed using intravenous administration of veterinary drugs "Zoletil ${ }^{\circledR} 100 "$ (Virbac, France) and "Xila" (Interchemie, the Netherlands) at doses of 5 and $2 \mathrm{mg} / \mathrm{kg}$, respectively.

Retinal imaging was performed using an indirect ophthalmoscope Reister All Pupil II (Rudolf Riester, Germany) and a PanRetinal 2.2 lens (Volk, USA). A laser focuser from the Alod-01 $810 \mathrm{~nm}$ laser device (Alcom Medica, Russia) was attached to the indirect ophthalmoscope. After determining the optic nerve, the focusing laser beam was moved to the desired position (about 1 diameter of the optic disc above and below the optic disc). Correct focusing was achieved by visualizing a clear contour of the laser pointer's focal spot against the background of the rabbit's fundus. Laser exposure to the eye retina was carried out until the appearance of a light coagulate, indicating a rupture of the Bruch membrane. The time of laser exposure before the appearance of the coagulate was recorded in the primary maps. On the retina of one eye of the animal, from 5 to 6 points were formed: 3 points each on the ventral part $(5,6,7 \mathrm{~h}$ relative to the dial) and the dorsal part $(11,12,1 \mathrm{~h})$. According to the literature data, the number of points in experimental works ranges from 3 to $9[9,11,12,13]$. 
In the study, three power options were tested: $500 \mathrm{~mW}$ (animal numbers 1.1-1.3, 2.1-2.3 left eye); $700 \mathrm{~mW}$ (animal numbers 1.4-1.6, 2.1-2.3 right eye); $1000 \mathrm{~mW}$ (animal numbers 2.4-2.6 left and right eye).

\subsection{Body weight registration}

The body weight of all animals was recorded before pathology induction to calculate the dose of drugs for anesthesia and then weekly using table scales (Table scales medical MT "Karapuz "(LLC "Modern technologies" Msk, Russia).

\subsection{Ophthalmoscopy}

Examination of the animal fundus was performed using a direct ophthalmoscope "VETA 200" (HEINE, Germany) and an indirect ophthalmoscope on the 1st (before pathology induction), 8th, 15th, 22nd, 29th days of the experiment. Fundus photography was performed using the SMARTSCOPE VET2 EY3 Optomed Oy (Ltd.) fundus camera (Finland).

\subsection{Pathomorphological examination}

\subsubsection{Histological examination}

After euthanasia, the animal eyes were enucleated. In animals with a model pathology of one eye, the intact eye was also removed in a separate histological glass. For histological examination, the material was fixed in Davidson's solution $(1.1-1.3,2.4)$ or formalin solution $10 \%(1.4-1.6,2.1-2.3,2.5,2.6)$, after that, the material was poured into paraffin. Tissue samples (the eye retina in the area of exposure to an ophthalmic laser) were cut out, dehydrated, soaked in paraffin, cut into sections, stained with hematoxylin and eosin, and examined by microscopy.

Histological preparations were analyzed using an Accu-Scope 3000 SERIES lightoptical microscope (USA) at magnifications of 40, 100, and 400. Microphotography was performed using a digital camera TOUPCAM UCMOS05100KPA (China) and software ToupView 3.7.7892.

\subsubsection{Immunohistochemical examination}

The IHC study was performed on the CD31 protein (a marker of intercellular contacts of endothelial cells) [7,13-15]. Eye samples were fixed in 10\% formalin (1.4-1.6, 2.1-2.3, 2.5, 2.6) or in Davidson's solution $(1.1-1.3,2.4)$ and subjected to standard histological laying. Sections with a thickness of 3-5 microns were made. Polylysine-coated slide glasses were used (BioVitrum, Russia). The sections were subjected to deparaffination and rehydration. Thermal antigen retrieval was performed in a boiling citrate buffer $\mathrm{pH}$ 6.0 (PraymBioMed, Russia) for 20 minutes. To block endogenous peroxidase, incubation with 3\% H2O2 was performed for 10 minutes. Mouse monoclonal antibodies to CD-31 (PraymBioMed, Russia) in a 1:75 dilution were used as primary antibodies. Incubation was carried out in a wet chamber at room temperature for 1 hour. Secondary multivalent antibodies Histofine Simple Stain MAX PO (MULTI) (Nichierei Biosciences Inc., Japan) and Histofine DAB-3s chromogen (Nichierei Biosciences Inc., Japan) were used as the imaging system. The secondary antibodies were incubated in a moist chamber at room temperature for 40 minutes. After incubation with each of the reagents, the glasses were washed three times in 
a pH 7.4 phosphate-salt buffer (PBS) (Rosmedbio, Russia). The working solution of chromogen was applied for 30 seconds, controlling the contrast of immunopositive staining (IS) with respect to the background under a microscope. Further, the sections were washed, stained with hematoxylin for 1 minute, subjected to dehydration and enlightenment, and enclosed under a cover glass. When setting up a positive control, rabbit kidney tissue was used. The stages of coloring the positive control were similar to those described above. When staining the negative antibody control, PBS was applied instead of the primary antibodies. The analysis was performed using a Carl Zeiss Axio Scope A1 microscope, an AxioCamICc 1 digital camera, and AxioVisionRel software. 4.8 (Germany). A semiquantitative assessment was performed in the area of the detected lesion in 3 fields of view at a magnification of 200. IS was evaluated in scores (from 0 to 5): 0 - absence of immunopositive cells; 1 - insignificant expression in immunopositive cells; 2 - most of the cells with weak expression; 3 - moderate expression in immunopositive cells; 4 immunopositive cells with moderate and pronounced expression; 5 - most of the cells with expressed marker expression. The number of vessels detected in the visual field was also counted.

\subsection{Data analysis}

Descriptive statistics were used for all the data: the data was checked for compliance with the normal distribution law. The test for compliance with the normal distribution law was carried out using the Shapiro-Wilk test. In the case of a normal distribution, the mean value and the standard error of the mean were calculated, which, together with the $n$ value, are presented in the final tables. In cases where the data did not correspond to normal distribution law, the median and quartile range were calculated. The inter-group differences were analyzed by parametric or nonparametric methods, depending on the type of distribution. To evaluate the data with signs of a normal distribution, an analysis of variance (ANOVA) was used, followed by an inter-group comparison (post hoc) using the Tukey's test (post hoc Tukey's). The intergroup differences for two samples obeying the normal distribution law were analyzed using the Student's criterion. And for data that do not obey the normal distribution law - the Kruskal-Wallis test with further pairwise comparison by the Mann-Whitney test in case of detection of a reliable influence of the studied factor.

Differences were determined at the 0.05 significance level. Statistical analysis was performed using licensed software GraphPad Prism 8.0 (GraphPad Software, USA).

\section{Research results and discussion}

\subsection{Laser coagulation}

According to the literature data [3-11], the model pathology is reproduced using three parameters: size of the planned coagulate, power of laser exposure and duration of its exposure, and exposure time does not exceed 0.2 seconds. In the experiment, the laser beam was focused manually, respectively, when the focus was blurred, the coagulate either did not appear, or had uneven edges, and the laser coagulation time could reach 3-4 seconds. The time data in the experiment significantly differ from the literature data in the direction of magnification, which may be due to manual focusing of the laser beam and its scattering when focusing incorrectly.

The laser exposure time is shown in Table 3. 
Table 3. Laser exposure time, sec, $\mathrm{M} \pm \mathrm{SEM}, \mathrm{n}=6$

\begin{tabular}{|c|c|c|c|c|}
\hline \multirow{2}{*}{ Animal numbers } & \multirow{2}{*}{$\begin{array}{c}\text { Power, } \\
\mathrm{mW}\end{array}$} & \multicolumn{2}{|c|}{ Part of the fundus } & \multirow{2}{*}{$\begin{array}{c}\text { Average time } \\
\text { for all impact } \\
\text { points }\end{array}$} \\
\cline { 3 - 4 } & & dorsal & ventral & \\
\hline $1.1-1.3,2.1-2.3$ left eye & 500 & $2.6 \pm 0.3$ & $1.8 \pm 0.5$ & $2.2 \pm 0.4$ \\
\hline $1.4-1.6,2.1-2.3$ right eye & 700 & $2.0 \pm 0.5$ & $2.3 \pm 0.9$ & $2.2 \pm 0.6$ \\
\hline $2.4-2.6$ left and right eye & 1000 & $1.4 \pm 0.2$ & $1.0 \pm 0.3$ & $1.2 \pm 0.1$ \\
\hline
\end{tabular}

The time data obeyed the normal distribution law. There were no statistically significant differences between the experimental groups (univariate ANOVA, $p>0.05$ ). At a power of $1000 \mathrm{~mW}$, less laser exposure time was required, at 20 of the 36 points/spots, the time was less than $1 \mathrm{~s}$. The maximum exposure time was at a power of $500 \mathrm{~mW}$, mainly up to 3-4 s. It was of an intermediate nature at a capacity of $700 \mathrm{~mW}$.

\subsection{Body weight}

These body weights corresponded to the normal distribution law. Primary data of group 1: $4313 \pm 185 \mathrm{~g}$, for group 2: $4118 \pm 196$. Analysis of the data using the Student's test (t-test) did not reveal statistically significant differences between the groups $(\mathrm{p}>0.05)$. Throughout the experiment, positive dynamics of body weight in animals in each group were recorded, but without significant differences ( $t$-test for dependent variables, $p>0.05$ ). Thus, modeling pathology in two or one eye did not affect the body weight of rabbits.

\subsection{Clinical observation}

The animals were monitored daily throughout the experiment. All animals were in a satisfactory condition, no signs of a change in the general condition towards depression or agitation, as well as blepharospasm and/or discharge from the conjunctival sac were recorded. Thus, modeling pathology in two or one eye did not affect the overall condition of the animals.

\subsection{Ophthalmoscopy}

The results of ophthalmoscopy before pathology induction corresponded to the norm for animals of this species. Immediately after laser coagulation, white depigmented slightly raised rounded burns were observed in all animals at all points of application.

\subsubsection{Results of ophthalmoscopy on the 8th day after pathology induction (500, 700 and $1000 \mathrm{~mW}$ )}

The color of the coagulates in the ventral and dorsal parts was different, so in the ventral part the spots had a white or gray center with a thin golden contour, while the dorsal spots had a dark center with a thick golden contour on the periphery. This description was typical for all laser exposure powers. Also, vessels were detected in the dorsal part at some points during direct ophthalmoscopy, more often vessels were found at powers of 700 and 1000 $\mathrm{mW}$ (Fig. 1).

3.4.2 Results of ophthalmoscopy on the 15th day after pathology induction (500, 700 and $1000 \mathrm{~mW}$ ) 
On the 15th day of the experiment, in most cases, the coagulates in the ventral and dorsal parts looked the same regardless of the affected power: a dark center and a light periphery. Vessels were detected in the dorsal part and partially in the ventral part during direct ophthalmoscopy (Fig. 1).

\subsubsection{Results of ophthalmoscopy on the 22nd day after pathology induction (700 and $1000 \mathrm{~mW}$ )}

The coagulants looked the same: a dark center with a thin light periphery. Some coagulants obtained at a power of $700 \mathrm{~mW}$ merged with the surrounding background. The vessels were rarely found only on the periphery, they were pale and thin (Fig. 1).

\subsubsection{Results of ophthalmoscopy on the 29th day after pathology induction (1000 $\mathrm{mW})$}

The coagulates had a gray center with a thin light periphery, and the vessels on the periphery were pale and thin (Fig. 1).

Thus, laser exposure at all power levels resulted in damage to the outer layers of the retina, which was most pronounced at a power of $1000 \mathrm{~mW}$. To model the pathology, it is necessary to choose the power that causes minimal damage in the outer layer, but affects the pigment layer and the Bruch membrane (no more than $700 \mathrm{~mW}$ ), which is consistent with the literature data [11].

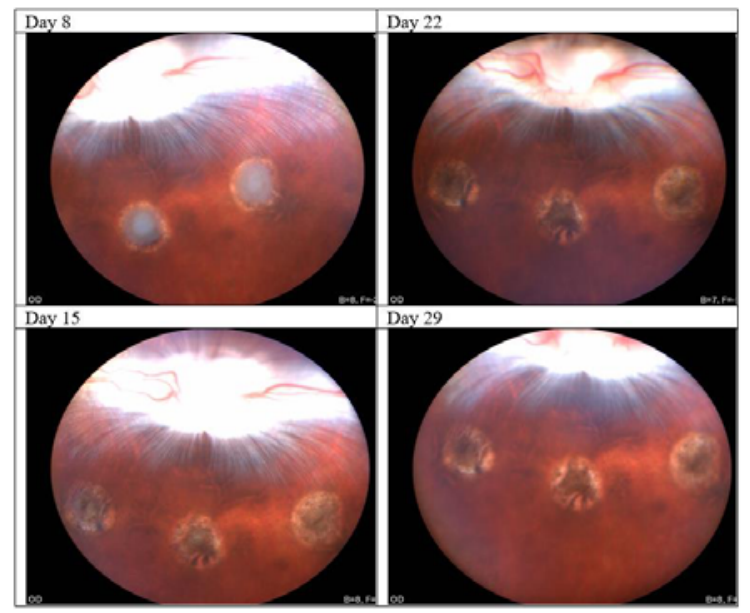

Fig.1. Ventral part of rabbit fundus 2.5 , power $1000 \mathrm{~mW}$

\subsection{Pathomorphological examinations}

\subsubsection{Histological examination}

Histological examination of the eye wall revealed lesions of varying intensity. In some cases, exposure caused necrosis of the entire vascular membrane up to the sclera (rabbit No. 2.4). With a decrease in the exposure intensity, the lesion depth decreased, as well as in the vascular layer, the pigment layer, and the layer of photosensory cells, vascular proliferation could be observed (Fig. 2), but with routine hematoxylin-eosin staining, the assessment of 
proliferation is difficult. With minimal exposure to an ophthalmic laser (at a power of 500 $\mathrm{mW}$ ), the pathological process mainly affected the vascular layer and the 1 st and 2nd layers of the retina, without causing massive necrosis, and at the same time stimulating vascular proliferation.

In all cases of material fixation in formalin (13 eyes), retinal detachment was detected (complete $n=11$ or partial $n=2$ ), and when fixed in Davidson's solution ( 5 eyes), partial detachment was detected only in 1 eye, the rest did not have this effect of fixation.

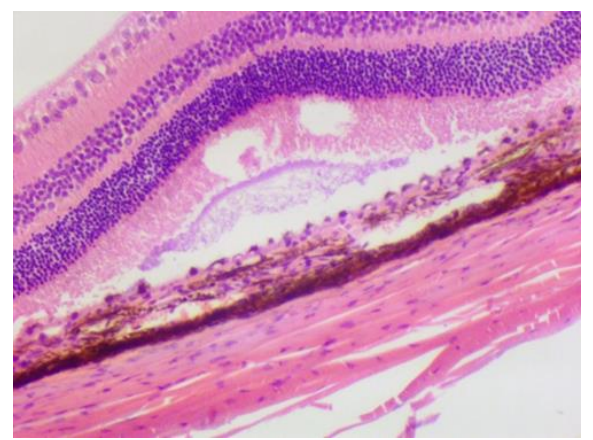

Fig. 2. Section of the left eye of the rabbit No. 1.2. The lesion area with pronounced edema, local thickening of the vascular layer with vascular proliferation. Fixative - Davidson's solution. Color hematoxylin eosin. Magnification x100. Power $500 \mathrm{~mW}$. 8th day after pathology induction

\subsubsection{Immunohistochemical examination}

Under microscopy, the number of vessels detected in the field of view in the affected area was not significantly less than in the intact eye, which can be associated with severe tissue damage by laser due to the increased exposure time compared to the literature data [3-11]. Further research is required to improve the method with a shorter exposure time. An IHC study of rabbit eyes revealed membrane-cytoplasmic immunopositive staining of vascular endotheliocytes. There was also a pronounced nonspecific binding of chromogen to retinal melanocytes.

The data on the intensity of CD31 staining (marker expression) and the number of vessels in the visual field did not follow the normal distribution law (Table 4). According to the results of statistical analysis of the data, there was no significant influence of the "group" factor (laser exposure power) on the indicators between the groups (Kruskal-Wallis test, $\mathrm{p}>0.05$ ). There was a tendency to increase the intensity of staining on the 15 th day after pathology induction (at a power of $500 \mathrm{~mW}$ ) and on the 29th day (at a power of 1000 $\mathrm{mW})$.

Table 4. Expression of CD31, Me(Q1;Q3)

\begin{tabular}{|c|c|c|c|c|c|c|}
\hline \multirow{2}{*}{ Animal numbers } & \multirow{2}{*}{$\begin{array}{l}\text { Pow } \\
\text { er, } \\
\text { mW }\end{array}$} & \multirow{2}{*}{$\begin{array}{l}\text { Day of the } \\
\text { experiment } \\
\text { after } \\
\text { pathology } \\
\text { induction }\end{array}$} & \multicolumn{2}{|c|}{ Staining intensity, scores } & \multicolumn{2}{|c|}{$\begin{array}{l}\text { Number of vessels detected } \\
\text { in the field of view }\end{array}$} \\
\hline & & & $\begin{array}{c}\text { in the field of } \\
\text { model pathology }\end{array}$ & $\begin{array}{c}\text { intact } \\
\text { eye }\end{array}$ & $\begin{array}{l}\text { in the field of } \\
\text { model pathology }\end{array}$ & $\begin{array}{c}\text { intact } \\
\text { eye }\end{array}$ \\
\hline $1.1-1.3,2.1,2.3 \mathrm{left}$ & 500 & 8th & $2(2 ; 3) n=5$ & \multirow{4}{*}{$\begin{array}{c}2(2 ; 4) \\
n=5\end{array}$} & $4(4 ; 5) n=5$ & \multirow{4}{*}{$\begin{array}{c}5(3 ; 5) \\
n=5\end{array}$} \\
\hline eye & & 15 th & $3(3 ; 3) n=6$ & & $2.5(2 ; 4) \mathrm{n}=6$ & \\
\hline $2.1,2.3$ right eye & 700 & 15 th & $2.5(2 ; 3) \mathrm{n}=6$ & & $4.5(3 ; 5) n=6$ & \\
\hline $\begin{array}{l}2.4 \text { left and right } \\
\text { eye, } 2.6 \text { left eye }\end{array}$ & 1000 & 29th & $3(2 ; 4) n=7$ & & $\begin{array}{c}3(2 ; 5) \\
n=7\end{array}$ & \\
\hline
\end{tabular}




\section{Conclusions}

1. Laserocaogulation of the rabbit retina can be performed by an indirect transpupillary technique using indirect ophthalmoscope and lens, however, the limitation of the method is the manual focusing of the laser beam, which requires a highly qualified researcher.

2. According to the results of ophthalmoscopy, neovascularization was observed on 8-15 days after laser coagulation and decreased by day 29 .

3. According to the results of histological examination, with minimal exposure to an ophthalmic laser $(500 \mathrm{~mW})$, indirect transpupillary laser coagulation does not cause massive tissue necrosis. According to IHC, there is a tendency to increase the intensity of CD31 staining (marker expression) at a power of $500 \mathrm{~mW}$ on the 15 th day after the pathology induction compared to the intact retina, which determines the desired power of no more than $500 \mathrm{~mW}$ for modeling choroidal neovascularization in rabbits using this method. Further research is needed to improve the method for the exposure time parameter to reduce the degree of tissue damage.

4. According to the results of the experiment, it is advisable to perform a histological examination of the retina and the vascular membrane of the rabbit's eyes when fixed in Davidson's solution.

5. Simulation of pathology in two or one eye did not affect the general condition of rabbits and the dynamics of body weight gain of animals, which indicates the safety of the method under study.

\section{References}

1. P. S. Bora et al, The Journal of Immunology, 174(1), 491 (2005)

2. S. J. Ryan, Trans Am Ophthalmol Soc., 77, 707 (1979)

3. R. S. Shah et al, Journal of Visualized Experiments, 106, e53502 (2015)

4. S. Narendran et al, Invest Ophthalmol Vis Sci., 61(10), 4 (2020)

5. L. Zhu et al, Communications Biology, 3(1), 1 (2020)

6. S. Inagaki et al, Current Neurovascular Research (2020)

7. J. H. Song et al, International journal of molecular sciences, 21(8), 2842 (2020)

8. X. Wang et al, International Journal of Ophthalmology, 13(3), 382 (2020)

9. O. Olvera-Montaño et al, Drug Design, Development and Therapy, 13, 3813 (2019)

10. R. Agrawal et al, Ocular immunology and inflammation, 28(1), 7 (2020)

11. J. Liu et al, International Journal of Nanomedicine, 14, 8819 (2019)

12. R. S. Shah et al, Journal of Visualized Experiments, 106, e53502 (2015)

13. J. L. Edelman, M. R. Castro, Experimental eye research, 71(5), 523 (2000)

14. S. Kang, S. K. Chung, Cornea, 29(2), 192 (2010)

15. X. Liu et al, Experimental and molecular pathology, 100(3), 451 (2016) 